\title{
Proficient blind users and mobile text-entry
}

\author{
Hugo Nicolau, Tiago Guerreiro, Joaquim Jorge, Daniel Gonçalves \\ IST / Technical University of Lisbon / INESC-ID \\ R. Alves Redol, 9, 1000-029 Lisbon, Portugal \\ \{hman, tjgv, jaj\}@ vimmi.inesc-id.pt, daniel.goncalves@inesc-id.pt
}

\begin{abstract}
Motivation - Understand how NavTap, an assistive text-entry method, stands in relation to traditional approaches.
\end{abstract}

Research approach - We performed a betweensubjects text-entry study with 12 blind users proficient with MultiTap and five blind users proficient with NavTap. Participants were asked to input ten sentences with different length and complexity.

Findings/Design - MultiTap significantly outperformed NavTap when considering text entry speed, confirming its theoretical advantage. However, when considering method effectiveness, NavTap's less experienced participants committed significantly fewer errors, indicating that it is both easier to learn and use, reaffirming it is an alternative for those unable to adjust to demanding adaptations.

Research limitations/Implications - Before NavTap appeared its users were unable to input text in a mobile device. Indeed, existing data make it difficult to assess differences between the users of either method. Further, NavTap users had less experience using the method (four months) than MultiTap (years).

Originality/Value - We contribute to understanding the limitations and merits of different text-entry approaches for blind people, after extensive usage.

Take away message - Different methods have different limitations and values. Selecting a match for a particular user may depend on his individual differences.

Keywords

Blind, Mobile, Text-Entry, Navtap, Multitap, Expert

\section{INTRODUCTION}

Mobile phones have become an important part of our daily life. They have altered our communication habits allowing us to contact or be contacted by anyone at any time almost anywhere. However, mobile phones are no longer mere communication devices. They have become constantly available personal devices for organizing and managing important data, e.g. scheduling tasks or storing personal documents and contacts. Mobile device interaction has evolved from the early, simpler devices, with basic communication capabilities and large buttons, to the recent stylish devices with complex applications and smaller keys or touch screens. Nevertheless some mobile phones are still (if not increasingly) too restrictive. Blind and visually impaired users face numerous difficulties due to these visually demanding interfaces.

Most blind people possess at least one cell phone (Guerreiro, 2008) and are able operate them at a basic level i.e. to perform and receive calls. Frequently, however, interaction is too complicated to take full advantage of the device and its applications. One of the major issues relates to text-entry, fundamental to a vast number of applications. This is particularly true for those users who were not capable to learn traditional mobile text-entry methods. This problem gains additional relevance when considering older or recently blind users that are likely to have a diminished development of the compensation mechanisms commonly associated with the blind population. Existing solutions assume a user with good orientation and memorization capabilities, but more than $82 \%$ of blind people are aged 50 years or older (WHO, 2008) and a great number have lost sight in an advanced stage of their life. Thus, there is a need for simpler and accessible text-entry methods that take into account these users' needs and capabilities.

In this paper, we evaluate how a tentative assistive textentry method, NavTap, places its users in opposition to the ones that are able to effectively input text with adapted traditional methods, i.e., MultiTap with screen readers. We aim to understand the differences between methods but also if an assistive approach is able to achieve efficiency and effectiveness levels that enable their users to accept their differences and maintain its use.

\section{BACKGROUND}

Text input on keypad based mobile phones is commonly achieved through a multi-tapping system where groups of 3 or 4 letters are assigned to each key. The most common text-entry method is MultiTap. Currently, the most common assistive solution for blind people uses this approach along with a screen reader (e.g. Nuance Talks), which replaces visual feedback with an audible representation. Nevertheless, the feedback is restricted to audio output and no information about letter placement on the keypad is available. This approach 


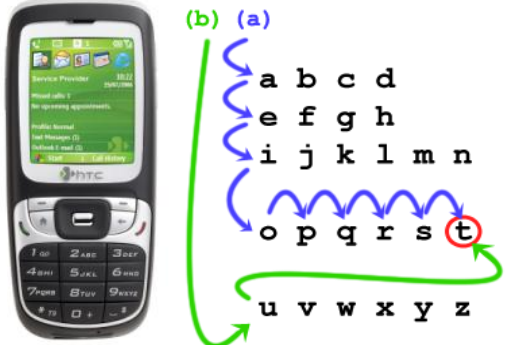

Figure 1. Right) Mobile device. Left) Vowel navigation: navigation scenarios.

forces the user to try to find the desired letter, where they often commit several errors in the process (Guerreiro, 2008) and can lead to situations where s/he simply gives up. This is especially true for those users that are neither tech-savvy nor comfortable exploring new functionalities.

The NavTap text-entry method (Guerreiro, 2008) tries to overcome these problems and ease the learning process by transforming letter selection into a navigation procedure. The alphabet is divided into five lines each starting with a vowel, as these are easy to recall. This alphabet representation can be navigated with a joystick (or direction keys) (Figure 1): horizontally through all letters and vertically through vowels. Both navigations are cyclical. Also, whilst navigating the alphabet users receive audio feedback on the current letter before accepting it. Contrast this with MultiTap where a key press can automatically lead to an error. In the worst case scenario, where the user does not have a good alphabet mental mapping, s/he can simply navigate straight forward (to the right) until s/he hears the desired letter. As illustrated in Figure 1, different navigation scenarios and expertise levels (e.g. one direction, two directions or four directions) can be achieved. There are no wrong buttons just shorter paths.

Besides the navigational buttons, the central key is used to accept the current letter or word (if no new letter is selected). When a word is entered it is read aloud. Other functions were placed in the corners and reference positions (i.e. ' 1 ', ' 3 ', and ' 5 ') as these are easy to find.

NavTap has been evaluated with blind users with reduced mobile experience (Guerreiro, 2008), who were only able to receive and perform calls. Results showed that they could learn and input text after a few minutes of practice. Moreover, NavTap showed that inexperienced users can input text effectively, outperforming the MultiTap method in a short learning period.

Recently, a longitudinal study was performed (Guerreiro, 2009) outside the laboratory setting with five participants. The main goals were to assess the method's effectiveness and learnability, as well as its influence on the users' daily lives. Again, the participants had reduced mobile experience. Nevertheless, the method was easy to learn and performance showed great improvements in real life scenarios. Indeed, users were able to control their mobile devices efficiently and changed both their communication patterns and mobile device usage.

\section{EVALUATION}

There is evidence that NavTap has a shorter learning curve than MultiTap (Guerreiro, 2008). The latter has shown to be hard to learn because it requires higher memorization capabilities and it is therefore error prone. Nevertheless, there are some blind users that are able to reach a high proficiency level with this method, although it can take several months or years of practice.

In this evaluation, we first want to analyze the performance level of a daily-use text-entry MultiTap user. We then want to assess NavTap's efficiency and effectiveness, using the MultiTap performance results as a baseline. Therefore, we will be able to analyze how users' experience, with both methods, influences their performance. This is relevant as it is vital to understand how an assistive method, theoretically less efficient, behaves after being used in real life scenarios.

Even though MultiTap has a theoretical advantage over NavTap, regarding the average number of required KeyStrokes Per Character (KSPC) (MacKenzie, 2007), there are no previously published results supporting this fact. In this evaluation we will observe if this advantage indeed occurs for expert users. Additionally, according to Silfverberg et al. (Silfverberg, 2000), MultiTap can support rates up to 25 or 27 Words Per Minute (WPM) for expert users. Nevertheless, to our knowledge, no input rates for expert blind users are documented.

\section{Procedure}

We used a between-subjects evaluation and each participant wrote ten different sentences in the Portuguese language. Moreover, sentences had 5 difficulty levels based on their lengths $(5,10,14,18$ and 24 characters). These lengths have no special justification other than enabling us to consistently vary the phrases difficulty levels.

Sentences were read aloud by the evaluation monitor and repeated by participants before entering them, thus assuring that participants understood them. They were asked to input text as quickly and correctly as possible. The sequence of sentences was randomized between participants, so the results would not be biased due to learning effects. In order to guarantee that participants would not suffer from fatigue effects, they could rest between trials.

Timing began when participants performed the first character of the sentence and ended when they performed the last keystroke. With this procedure the resulting sentences could contain errors. Thus, another of our goals was to observe the quality of the entered phrases with both methods. Also, participants could correct errors by deleting characters. The sentence completion time incorporates the time to correct those errors. Finally, in order to offer a more natural, familiar and realistic scenario, participants used their own mobile devices to enter the proposed sentences. 


\section{Participants}

This evaluation was performed in a formation centre for visually impaired people and all participants were trainees there. We found two types of users: those who had learned MultiTap (12 users) and those who could not (5 users). While NavTap has shown to be an alternative solution to those who could not learn MultiTap (Guerreiro, 2008), we have no knowledge about how both methods behave for experts.

In this evaluation we assess performance after long-term daily use. Therefore, we selected participants that had sent at least 5 text messages per week for the past 4 months, and were able to manage (add, remove and search) their contact list. The NavTap group was composed of 5 participants, 3 females and 2 males, with ages between 44 and 61 years old. These participants had not been able to learn the MultiTap approach. In fact, NavTap was the first mobile text-entry method that those participants were able to learn and use on a daily basis. For the past 4 months, they were able to fully control their mobile devices (Guerreiro, 2009). On the other hand, the MultiTap group was composed of 12 participants, 9 males and 3 females, with ages between 20 and 58 years old. The target group had an average mobile experience of 6.75 years.

\section{Results}

In order to achieve a better understanding of the obtained results, we must emphasize the differences in participants' profiles between text-entry methods. Firstly, NavTap participants were not able to learn the MultiTap method. Secondly, although being considered experienced (4 months usage), they have a much lower level of experience than MultiTap participants (an average usage of 6.75 years). Thus, our goal with was to observe what performance level this population can reach when compared to MultiTap's expert participants.

\section{Keystrokes per Character}

KeyStrokes Per Character is the number of keystrokes required, on average, to generate each character in a given language using a given text-entry technique (MacKenzie, 2007).

One of our goals with this evaluation was to analyze if MultiTap's theoretical advantage indeed occurs with expert users. The average Keystrokes Per Character rate

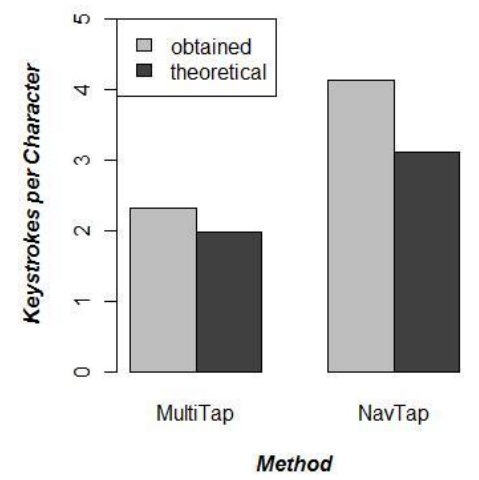

Figure 2. Keystrokes per character by text-entry method. was 2.31 for MultiTap, and 4.14 for NavTap (Figure 2). Indeed, MultiTap requires significant less KSPC than NavTap (one-tailed Mann-Whitney test, $p<0.01$ ). Also, MultiTap is closer to theoretical values (1.97 KSPC) than NavTap best case scenario (3.11 KSPC). The performance level of NavTap's participants is between the 2-way (using two directions) and 4-way (best case) navigational approaches.

\section{Text-Entry Speed}

In order to quantify text-entry speed we used the standard Words Per Minute measure (MacKenzie, 2007). Usually, this is calculated as (characters per second) $* 60 / 5$. As timing in our experiment started only after entering the first character, that character is not included in entry speed calculations. Hence, the phrase length is $\mathrm{n}-1$ characters in our computations.

As a consequence of a lower KSPC, MultiTap also outperformed NavTap regarding the words per minute input rate. The average text-entry speeds were 6.64 WPM for MultiTap and 3.82 WPM for NavTap. A onetailed Mann-Whitney test $(p<0.05)$ showed that the MultiTap group was significantly faster than NavTap's.

\section{Minimum String Distance Error Rate}

Recall that our procedure did not force participants to enter phrases correctly. As such, the text-entry speed was influenced by the quality of the transcribed phrases as participants may have entered more or less characters. Nevertheless, it is still helpful to observe the Minimum String Distance (MSD) error rate (MacKenzie, 2007) for both methods.

There was not a significant main effect (two-tailed Mann-Whitney test $p=0.1$ ) on MSD error rate; however, NavTap outperformed MultiTap with $0.85 \%$ against $2.08 \%$, respectively (Figure 3 ). Although a minor effect was found on MSD error rate, sentences transcribed by the NavTap group had a higher quality than MultiTap's.

\section{Error Rate}

As we said before, transcribed phrases could have some errors. However, participants were free to correct them during the evaluation. W For this experiment we considered an error as a character deletion. NavTap showed to be significantly less error prone than

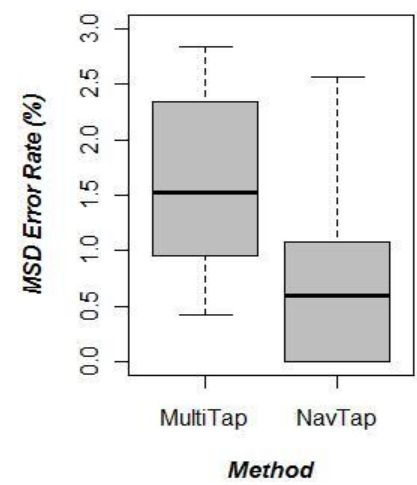

Figure 3. MSD error rate by text-entry method. 


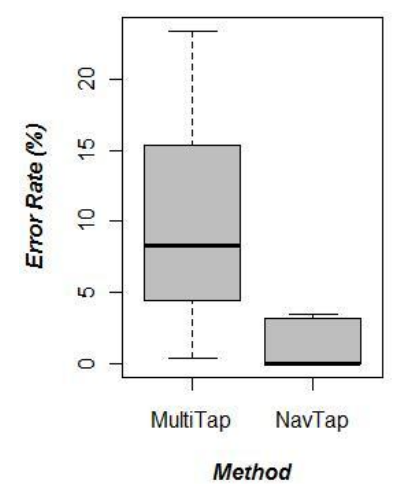

Figure 4. Error rate by text-entry method.

MultiTap (one-tailed Mann-Whitney test $p<0.01$ ). The average error rates were $10.03 \%$ for MultiTap and $1.32 \%$ for NavTap (Figure 4).

Furthermore, relating these results with MSD error rate, we can see that NavTap's sentences had a higher quality and a lower number of character deletions. Conversely, MultiTap's sentences were of poorer quality and had a higher error rate.

\section{CONCLUSIONS AND FUTURE WORK}

Some blind users are not able to learn traditional MultiTap approaches and thus unable to take full advantage of their mobile devices. As an alternative, NavTap has shown to be an easy to learn text-entry method for novice users (Guerreiro, 2008). The main goals of this evaluation were to assess the limitations and main advantages of both methods with expert users.

Indeed MultiTap's theoretical advantage occurs for both KeyStrokes per Character and, consequently, Words per Minute metrics, meaning higher efficiency. These results were somewhat expected as expert users can take full advantage of a given technique. Being MultiTap a more complex method than NavTap, it enables higher text-entry rates. However, the obtained results are still far from the ones reported by Silfverberg et al. (Silfverberg, 2000) for expert users. This may indicate that a simple substitution of visual feedback by audio feedback (i.e. a screen reader) may hinder the learning process. Although sighted experienced users are likely to input text at astonishing rates, during their long-term experience (months or years) they were able to receive proper feedback on their evolution. We believe this learning process to be what differences blind and sighted expert users the most. Further research is needed to understand the major implications of the visual feedback absence and enrich audio representation to overcome the flaws.

On the other hand, NavTap outperforms MultiTap regarding output quality and produces significantly fewer errors. The small number of errors indicates that NavTap is indeed more effective and easier to use. This fact gains higher relevance because NavTap's participants had a lower experience level (less time using the method). Indeed, NavTap was the only mobile text-entry method they were capable to learn and use in a daily basis, much due to the reduced number of produced errors. Results indicate that both methods have different target audiences. On one hand, MultiTap has shown to be a more efficient method with higher text-entry rates. On the other hand, NavTap is an easy to learn and effective technique that has show to be a suitable alternative for those users who were not able to learn MultiTap.

We believe that this paper provides a good basis for future work. Particularly, we intend to better understand why NavTap participants were not able to learn MultiTap and characterize both user groups in terms of cognitive and physical capabilities. Then we will be able to objectively state the differences between those two groups and relate them with text-entry performance.

There is a need to identify and comprehend the blind population individual characteristics and their implications for mobile text-entry methods future designs. From our experience, some users are unable to learn current text-entry techniques, revealing an inadequacy of mobile interfaces to their capabilities. This is particularly visible on elder and adventitious blind users, which require simpler text-entry methods.

\section{ACKNOWLEDGMENTS}

This work was supported by FCT (INESC-ID multiannual funding) through the PIDDAC Program funds. Hugo Nicolau and Tiago Guerreiro were supported by FCT, grants SFRH/BD/46748/2008 and SFRH/BD/28110/2006, respectively.

\section{REFERENCES}

Guerreiro, T., Lagoá, P., Santana, P., Nicolau, H., and Jorge, J. (2008). Mobile Text-Entry Models for People with Disabilities. European Conference on Cognitive Ergonomics 2008, Madeira, Portugal.

Guerreiro, T., Nicolau, H., Jorge, J. and Gonçalves, D. (2009). NavTap: a Long Term Study with Excluded Blind Users. In Proc. of SIG ASSETS, AMC Press.

MacKenzie, I. S., and Tanaka-Ishii, K. (2007). Text Entry Systems: Mobility, Accessibility, Universality. Morgan Kaufmann Publishers Inc.

Silfverberg, M., MacKenzie, I.S, and Korhonen, P. (2000). Predicting text entry speed on mobile phones. In Proc. SIG CHI, ACM Press.

World Health Organization (2008). Magnitude and causes of visual impairment.

http://www.who.int/mediacentre/factsheets/fs282/ (Last Visited on 29/03/2010) 